\title{
MONOTONE UNITARY FAMILIES
}

\author{
DANIEL GRIESER
}

(Communicated by Richard Rochberg)

\begin{abstract}
A unitary family is a family of unitary operators $U(x)$ acting on a finite-dimensional Hermitian vector space, depending analytically on a real parameter $x$. It is monotone if $\frac{1}{i} U^{\prime}(x) U(x)^{-1}$ is a positive operator for each $x$. We prove a number of results generalizing standard theorems on the spectral theory of a single unitary operator $U_{0}$, which correspond to the 'commutative' case $U(x)=e^{i x} U_{0}$. So these may be viewed as a noncommutative generalization of the spectral theory of $U_{0}$. Also, for a two-parameter unitary family (for which there is no analytic perturbation theory) we prove an implicit function type theorem for the spectral data under the assumption that the family is monotone in one argument.
\end{abstract}

\section{INTRODUCTION}

Let $U(x)$ be a family of unitary operators on a Hermitian vector space $V$ of dimension $M<\infty$, depending real analytically on $x \in \mathbb{R}$ (or an interval in $\mathbb{R}$ ). Then

$$
D(x):=\frac{1}{i} U^{\prime}(x) U(x)^{-1}
$$

is symmetric. Here $U^{\prime}(x)$ is the derivative with respect to $x$. We call $U$ monotone if $D(x)$ is a positive operator for all $x$. Denote

$$
W(x)=\operatorname{Ker}(I-U(x)) \quad \text { and } \quad \mathcal{Z}=\{x: W(x) \neq\{0\}\} .
$$

Thus $x \in \mathcal{Z}$ iff $U(x)$ has eigenvalue one.

A model case for this setup is $U(x)=e^{i x} U_{0}$ for a unitary $U_{0}$. Then $W(x)$ is the eigenspace of $U_{0}$ with eigenvalue $e^{-i x}$. Standard facts from the spectral theory of $U_{0}$ may be restated in terms of $\mathcal{Z}$ and $W(x)$, for example:

- $\mathcal{Z}$ is a $2 \pi$-periodic sequence, having exactly $M$ terms in each half-open interval of length $2 \pi$ (counting 'multiplicities').

- If $I$ is an interval of length less than $2 \pi$, then the spaces $W(x), x \in I$, are linearly independent (even pairwise orthogonal).

- If $\left\|\left(I-U\left(x_{0}\right)\right) \varphi\right\| \leq \varepsilon\|\varphi\|$ for some $\varphi \in V \backslash\{0\}, x_{0} \in \mathbb{R}$ and $\varepsilon \geq 0$, then $e^{-i x_{0}}$ lies within distance $\varepsilon$ of an eigenvalue of $U_{0}$ (and so $\operatorname{dist}\left(x_{0}, \mathcal{Z}\right) \leq$ $\pi \varepsilon / 2)$. Furthermore, if $\varepsilon^{\prime}>\varepsilon$ and $P$ denotes the orthogonal projection to $\bigoplus_{x:\left|e^{-i x}-e^{-i x_{0}}\right|<\varepsilon^{\prime}} W(x)$, then

$$
\|\varphi-P \varphi\| \leq \frac{\varepsilon}{\varepsilon^{\prime}}\|\varphi\| \text {. }
$$

Received by the editors August 2, 2011.

2010 Mathematics Subject Classification. Primary 47A55; Secondary 47A56, 15A22.

Key words and phrases. Perturbation theory, spectral theory. 
In this paper we prove generalizations of these facts to arbitrary monotone unitary families; see Theorem 1 in Section 2 and Theorems 2 and 3 in Section 3. The estimates are expressed in terms of uniform bounds on the first and second derivatives of $U$ : Assume $d_{\min }, d_{\max }, d_{2}>0$ are such that

$$
d_{\min } I \leq D(x) \leq d_{\max } I, \quad\left\|U^{\prime \prime}(x)\right\| \leq d_{2} \quad \text { for all } x .
$$

Of course, such constants always exist locally. However, in applications of our results, see [2], it is essential that the estimates are uniform in terms of (3), and do not depend on additional data such as the separation of elements of $\mathcal{Z}$; see the explanation below.

An important difference between a general unitary family and the model case is that $U(x)$ and $U\left(x^{\prime}\right)$ do not in general commute for $x \neq x^{\prime}$. A consequence of this is that while one may take a logarithm of $U$, i.e. find an analytic family $A(x)$ of symmetric operators such that

$$
U(x)=e^{i A(x)} \quad \text { for all } x,
$$

it is usually not true that $D(x)$ equals $A^{\prime}(x)$, or even that positivity of $D(x)$ implies positivity of $A^{\prime}(x)$. The opposite implication is true, however. See Section 4

Finally, we prove a result on two-parameter perturbation theory. Recall the main result of one-parameter perturbation theory: For an analytic family $U(x)$ of unitary operators on $V$, there are real analytic functions $\lambda_{j}(x), \varphi_{j}(x)$ for $j=1, \ldots, M=$ $\operatorname{dim} V$ having values in the unit circle $\mathbb{T}$ of $\mathbb{C}$ and in $V$, respectively, such that for each $x$ the eigenvalues of $U(x)$ are $\lambda_{j}(x)$, with corresponding orthonormal basis of eigenvectors $\varphi_{j}(x)$ (see [4], [3, Remark II.6.4]). Since $\mathbb{R}$ is simply connected, the maps $\mathbb{R} \rightarrow \mathbb{T}, x \mapsto \lambda_{j}(x)$ can be lifted to real analytic maps $\mathbb{R} \rightarrow \mathbb{R}, x \mapsto \mu_{j}(x)$ satisfying $\lambda_{j}(x)=e^{i \mu_{j}(x)}$ for each $x$ and $j$. So we have

$$
U(x) \varphi_{j}(x)=e^{i \mu_{j}(x)} \varphi_{j}(x) .
$$

It is well-known that the analogous statement for two-parameter families of operators is false in general. However, we prove a related implicit function type theorem for the spectral data of a two-parameter family for which the dependence on one parameter is monotone. It may be regarded as the natural unitary family generalization of the one-parameter perturbation theory. See Theorem 7 in Section 5.

The analytic functions $\mu_{j}$ and $\varphi_{j}$ play a central role in the proofs of our theorems. It is essential to control their derivatives. For $\mu_{j}$ this is easy from (3); see (9). However, $\varphi_{j}$ may vary wildly whenever $e^{i \mu_{j}}$ is very close to another eigenvalue. To control this variation is the main technical problem in the proof of the generalization of (2), Theorem 3. Note that, unlike in the model case, one may not assume that the $e^{i \mu_{j}(x)}$ for fixed $x$ but varying $j$ are uniformly separated, or equivalently that the elements of $\mathcal{Z}$ are uniformly separated. This can already be seen in the simple example $U(x)=e^{i x L}$, where $L$ is a diagonal matrix with positive diagonal entries that are independent over $\mathbb{Q}$ : If $M \geq 2$, then for any $\varepsilon>0$ there are $x, x^{\prime} \in \mathcal{Z}$ satisfying $0<\left|x-x^{\prime}\right|<\varepsilon$.

The problems we study here address some very basic questions of a spectral theoretic nature which seem not to have been studied in the literature. They arose in the context of a singular perturbation problem: In 2 we study the eigenvalues and eigenfunctions of the Laplacian on a space $X^{N}$ which has a fixed compact part 
connected by cylindrical necks of length $N>0$, and in particular their asymptotic behavior as $N \rightarrow \infty$. The unitary families arise from the scattering matrix of the limit problem (infinitely long 'necks').

\section{Eigenvalue Distribution}

Theorem 1. Let $U$ be a monotone unitary family on $\mathbb{R}$. Then $\mathcal{Z} \subset \mathbb{R}$ is a discrete subset, and more precisely for all $A<B$,

$$
\left|\sum_{x: A<x<B} \operatorname{dim} W(x)-\frac{1}{2 \pi} \int_{A}^{B} \operatorname{tr} D(x) d x\right|<M(:=\operatorname{dim} V) .
$$

In the special case $U(x)=e^{i x L} U_{0}, L>0$, this implies the asymptotics

$$
\sum_{x: 0<x<B} \operatorname{dim} W(x) \sim \frac{\operatorname{tr} L}{2 \pi} B, \quad B \rightarrow \infty .
$$

This is the Weyl asymptotics of a quantum graph; see for example 11. We give a much simpler proof than 1 .

First, we differentiate (5) and obtain monotonicity of the functions $\mu_{j}$ from monotonicity of $U$ :

$$
\mu_{j}^{\prime}=\left(\frac{1}{i} U^{\prime} U^{-1} \varphi_{j}, \varphi_{j}\right)=\left(D \varphi_{j}, \varphi_{j}\right)>0 .
$$

Also, for each $x$ we have

$$
W(x)=\operatorname{span}\left\{\varphi_{j}(x): \mu_{j}(x) \in 2 \pi \mathbb{Z}\right\} .
$$

Proof of Theorem 1. We have

$$
\begin{aligned}
\sum_{j}\left(\mu_{j}(B)-\mu_{j}(A)\right) & =\int_{A}^{B} \sum_{j}\left\langle D(x) \varphi_{j}(x), \varphi_{j}(x)\right\rangle d x \\
& =\int_{A}^{B} \operatorname{tr} D(x) d x .
\end{aligned}
$$

Since the $\mu_{j}$ are strictly increasing, we get from (8),

$$
\begin{aligned}
\sum_{x: A<x<B} \operatorname{dim} W(x) & =\sum_{j} \#\left\{k \in \mathbb{Z}: \mu_{j}(A)<2 \pi k<\mu_{j}(B)\right\} \\
& =\sum_{j}\left(\frac{\mu_{j}(B)-\mu_{j}(A)}{2 \pi}+R_{j}\right)
\end{aligned}
$$

with $\left|R_{j}\right|<1$, and this gives (6).

\section{Eigenspaces}

In this section we consider monotone unitary families satisfying the estimates (3). By (7) we have

$$
d_{\min } \leq \mu_{j}^{\prime}(x) \leq d_{\max } \quad \text { for all } x \text { and } j=1, \ldots, M .
$$

First, we have independence of eigenspaces. 
Theorem 2. Let $U$ be a monotone unitary family satisfying (31). Let $I$ be an interval of length at most $\frac{2 d_{\min }}{d_{2} M}$. Then the spaces $W(x), x \in I$, are independent, i.e.:

$$
\text { If } \varphi_{x} \in W(x) \text { for each } x \in I \cap \mathcal{Z} \text { and } \sum_{x} \varphi_{x}=0 \text {, then } \varphi_{x}=0 \forall x .
$$

The following theorem gives a stable version of almost orthogonality.

Theorem 3. Let $U$ be a monotone unitary family satisfying (3). Assume $\varphi \in V \backslash 0$ satisfies

$$
\left\|\left(I-U\left(x_{0}\right)\right) \varphi\right\| \leq \varepsilon\|\varphi\| .
$$

Then

$$
\operatorname{dist}\left(x_{0}, \mathcal{Z}\right) \leq \frac{\pi}{2} \frac{\varepsilon}{d_{\min }} .
$$

Furthermore, there is a constant $C$ only depending on $d_{\text {min }}, d_{\text {max }}, d_{2}, M$ such that the following holds: Suppose $0<\varepsilon<\varepsilon^{\prime}<C^{-1}$ and $\varepsilon / \varepsilon^{\prime}<C^{-1}$. Denote by $P_{W}$ the orthogonal projection to $W=\bigoplus_{\left|x-x_{0}\right| \leq \varepsilon^{\prime}} W(x)$. Then

$$
\left\|\varphi-P_{W} \varphi\right\| \leq C\left(\frac{\varepsilon}{\varepsilon^{\prime}}\right)^{\frac{1}{M+1}}\|\varphi\| .
$$

In the proofs of Theorems 2 and 3 we will need the following estimate, which replaces orthogonality of the eigenspaces of a unitary operator.

Lemma 4. If $\varphi \in W(x), \psi \in W(y)$ and $x \neq y$, then

$$
|\langle D(x) \varphi, \psi\rangle| \leq \frac{d_{2}}{2}|x-y| \cdot\|\varphi\| \cdot\|\psi\| .
$$

Proof. By Taylor's formula,

$$
U(y) U(x)^{-1}=I+(y-x) U^{\prime}(x) U(x)^{-1}+(y-x)^{2} R, \quad\|R\| \leq \frac{d_{2}}{2},
$$

so if $U(x) \varphi=\varphi, U(y) \psi=\psi$, then

$$
\begin{aligned}
\langle\varphi, \psi\rangle & =\left\langle U(x)^{-1} \varphi, U(y)^{-1} \psi\right\rangle=\left\langle U(y) U(x)^{-1} \varphi, \psi\right\rangle \\
& =\langle\varphi, \psi\rangle+i(y-x)\langle D(x) \varphi, \psi\rangle+(y-x)^{2}\langle R \varphi, \psi\rangle,
\end{aligned}
$$

and this gives (14).

Proof of Theorem 2. Let $\varphi_{x} \in W(x)$ for $x \in I \cap \mathcal{Z}$, and assume $\sum_{x} \varphi_{x}=0$. Let $\varphi_{x_{0}}$ have maximal norm among all $\varphi_{x}$. Then $0=\left\langle D\left(x_{0}\right) \varphi_{x_{0}}, \sum_{x} \varphi_{x}\right\rangle$ gives with (3) and (14):

$$
\begin{aligned}
d_{\min }\left\|\varphi_{x_{0}}\right\|^{2} & \leq\left\langle D\left(x_{0}\right) \varphi_{x_{0}}, \varphi_{x_{0}}\right\rangle=\left|\sum_{x \neq x_{0}}\left\langle D\left(x_{0}\right), \varphi_{x_{0}}, \varphi_{x}\right\rangle\right| \\
& \leq \sum_{x \neq x_{0}} \frac{d_{2}}{2}|I| \cdot\left\|\varphi_{x_{0}}\right\| \cdot\left\|\varphi_{x}\right\| \leq(M-1) \frac{d_{2}}{2}|I| \cdot\left\|\varphi_{x_{0}}\right\|^{2},
\end{aligned}
$$

so if $d_{\text {min }}>(M-1) \frac{d_{2}}{2}|I|$, then $\varphi_{x_{0}}=0$ and hence $\varphi_{x}=0$ for all $x$. This implies the claim. 
Proof of Theorem 3. The first estimate follows easily from the fact that, by the lower bound in (9), an eigenvalue close to one of $U\left(x_{0}\right)$ will turn into an eigenvalue equal to one of $U(x)$, for some $x$ close to $x_{0}$ : Let $B(x)=I-U(x)$ and let $\lambda_{j}(x)=$ $1-e^{i \mu_{j}(x)}$ be the eigenvalues of $B(x)$. The assumption (11) implies that $\left|\lambda_{j}\left(x_{0}\right)\right| \leq \varepsilon$ for some $j$, and this implies that $\operatorname{dist}\left(\mu_{j}\left(x_{0}\right), 2 \pi \mathbb{Z}\right) \leq \frac{\pi}{2} \varepsilon$, and then $\mu_{j}^{\prime} \geq d_{\text {min }}$ shows that there is an $x$ satisfying $\left|x-x_{0}\right|<\pi \varepsilon / 2 d_{\min }$ and $\mu_{j}(x) \in 2 \pi \mathbb{Z}$; hence $x \in \mathcal{Z}$, so (12) follows.

For $\delta>0$ let $P_{\delta}(x)$ denote the orthogonal projection to the sum of the eigenspaces of $B(x)$ with eigenvalues $\left|\lambda_{j}(x)\right| \leq \delta$. Then $\left\|B\left(x_{0}\right) \varphi\right\| \leq \varepsilon\|\varphi\|$ implies that

$$
\left\|\varphi-P_{\delta}\left(x_{0}\right) \varphi\right\| \leq \frac{\varepsilon}{\delta}\|\varphi\|
$$

(see (2), which also applies to normal operators). To make this a good estimate, we want to take $\delta \gg \varepsilon$. Our goal is to replace $P_{\delta}\left(x_{0}\right)$ by $P_{W}$ here. The idea is that eigenspaces of $B\left(x_{0}\right)$ with eigenvalue $\left|\lambda_{j}\left(x_{0}\right)\right| \leq \delta$ will turn into nullspaces of $B(x)$ for some $x$ within $2 \delta / d_{\min }$ of $x_{0}$ by the first part of this proof. However, the variation of eigenspaces is much less well behaved than the variation of eigenvalues: An eigenspace may change rapidly with $x$ if the eigenvalue is very close to another eigenvalue. Therefore, we need to consider not single eigenspaces but rather clusters of eigenspaces.

The variation of eigenspaces is given as follows (see [3]): Fix $x$. If $B(x)$ has no eigenvalue on the circle $|\lambda|=\delta$, then, with a prime denoting derivative in $x$,

$$
P_{\delta}^{\prime}=\sum_{j:\left|\lambda_{j}\right|<\delta} \sum_{k:\left|\lambda_{k}\right|>\delta} \frac{1}{\lambda_{j}-\lambda_{k}}\left(P_{j} B^{\prime} P_{k}+P_{k} B^{\prime} P_{j}\right) .
$$

Here, all quantities are evaluated at $x$, and $P_{j}$ is the orthogonal projection to $\operatorname{span} \varphi_{j}$. Taking norms and using orthogonality of the $P_{j}$ one obtains from this, using $\left\|B^{\prime}\right\| \leq d_{\max }$,

$$
\left\|P_{\delta}^{\prime}\right\| \leq d_{\max } M\left(\min _{\left|\lambda_{j}\right|<\delta,\left|\lambda_{k}\right|>\delta}\left|\lambda_{j}-\lambda_{k}\right|\right)^{-1} .
$$

We need to choose $\delta$ carefully to make the spectral gap not too small: Let $s=$ $\left(\varepsilon^{\prime} / \varepsilon\right)^{1 /(M+1)}$ and consider the $M$ disjoint subintervals

$$
\left[\varepsilon s^{k}, \varepsilon s^{k+1}\right) \quad \text { for } k=1, \ldots, M
$$

of $\left(\varepsilon, \varepsilon^{\prime}\right)$. Since $B\left(x_{0}\right)$ has $M$ eigenvalues and one of them has absolute value $\leq \varepsilon$, at least one of these intervals contains no $\left|\lambda_{j}\left(x_{0}\right)\right|$. Assume

$$
[\delta, \delta s), \delta=\varepsilon s^{k}, \text { contains no }\left|\lambda_{j}\left(x_{0}\right)\right| \text {. }
$$

We then have:

A) The eigenvalues of $B\left(x_{0}\right)$ with $\left|\lambda_{j}\left(x_{0}\right)\right| \leq \delta$ are in $1-1$ correspondence with those $x \in \mathcal{Z}$ (counted with multiplicity $\operatorname{dim} W(x))$ satisfying

$$
\left|x-x_{0}\right| \leq \delta^{\prime}:=\frac{2 \delta}{d_{\min }} .
$$

(Proof: Each such eigenvalue turns into a zero of $\lambda_{j}(x)$ for such an $x$, by the argument at the beginning of this proof. Conversely, if $\lambda_{j}(x)=0$, then $\left|\lambda_{j}\left(x_{0}\right)\right| \leq\left|x-x_{0}\right| d_{\max }$ since $\left|\lambda_{j}^{\prime}\right|=\mu_{j}^{\prime} \leq d_{\max }$ and hence $\left|\lambda_{j}\left(x_{0}\right)\right| \leq 2 \delta \frac{d_{\max }}{d_{\min }}<\delta s$ provided $\varepsilon / \varepsilon^{\prime}$ is sufficiently small (and therefore $s$ big), and by (18) this implies further that $\left|\lambda_{j}\left(x_{0}\right)\right|<\delta$.) 
B) The smaller interval $\left(\delta+2 \delta \frac{d_{\max }}{d_{\min }}, \delta s-2 \delta \frac{d_{\max }}{d_{\min }}\right)$ contains no $\left|\lambda_{j}(x)\right|$ for any $x$ with $\left|x-x_{0}\right| \leq \delta^{\prime}$. (This follows directly from $\left|\lambda_{j}^{\prime}\right| \leq d_{\max }$.)

The length of the interval in $\mathrm{B})$ is $\delta\left(s-1-4 \frac{d_{\max }}{d_{\min }}\right)$, which is $\geq \delta s / 2>0$ if $s$ is sufficiently big. Choose $\tilde{\delta}$ in this interval. Then we get from (17) $\left\|P_{\tilde{\delta}}^{\prime}(x)\right\| \leq$ $d_{\max } M(\delta s / 2)^{-1}$ for $\left|x-x_{0}\right| \leq \delta^{\prime}$. Integration gives

$$
\left\|P_{\tilde{\delta}}(x)-P_{\tilde{\delta}}\left(x_{0}\right)\right\| \leq \varepsilon_{1}:=\frac{4}{s} \frac{d_{\max }}{d_{\min }} M \quad \text { for }\left|x-x_{0}\right| \leq \delta^{\prime} .
$$

This implies that

$$
\left\|\psi-P_{\delta}\left(x_{0}\right) \psi\right\| \leq \varepsilon_{1}\|\psi\| \quad \text { for } x \in \mathcal{Z},\left|x-x_{0}\right| \leq \delta^{\prime}, \psi \in W(x)
$$

since then $P_{\tilde{\delta}}(x) \psi=\psi$ and $P_{\tilde{\delta}}\left(x_{0}\right)=P_{\delta}\left(x_{0}\right)$.

Next we want to extend this estimate to $\psi \in W^{\prime}:=\bigoplus_{\left|x-x_{0}\right| \leq \delta^{\prime}} W(x)$. For this it is essential that by (14) and the positive definiteness of $D(x)$, the angles between different $W(x)$ are bounded away from zero. To carry this out, we first derive from (14) an estimate where all $D(x)$ are replaced by $D\left(x_{0}\right)$ : From $D^{\prime}=U^{\prime \prime} U^{-1}+D^{2}$ we have $\left\|D^{\prime}\right\| \leq d_{2}+d_{\max }{ }^{2}$; integration yields $\left\|D\left(x_{0}\right)\right\| \leq\|D(x)\|+\left(d_{2}+d_{\max }{ }^{2}\right) \delta^{\prime}$ for $\left|x-x_{0}\right| \leq \delta^{\prime}$, and then (14) gives, with $D_{0}:=D\left(x_{0}\right)$,

$$
\left|\left\langle D_{0} \psi_{x}, \psi_{y}\right\rangle\right| \leq \delta^{\prime \prime}\left\|\psi_{x}\right\| \cdot\left\|\psi_{y}\right\|, \quad \delta^{\prime \prime}:=\delta^{\prime}\left(d_{2}+d_{\max }^{2}+d_{2}\right)
$$

for $\psi_{x} \in W(x), \psi_{y} \in W(y), x \neq y$ and $\left|x-x_{0}\right| \leq \delta^{\prime},\left|y-x_{0}\right| \leq \delta^{\prime}$. Introduce the scalar product $(\varphi, \psi)_{D_{0}}:=\left\langle D_{0} \varphi, \psi\right\rangle$ on $V$, with norm $\|\psi\|_{D_{0}}=\sqrt{\left\langle D_{0} \psi, \psi\right\rangle}$. Then

$$
d_{\min }\|\psi\|^{2} \leq\|\psi\|_{D_{0}}^{2} \leq d_{\max }\|\psi\|^{2},
$$

so (21) gives

$$
\left|\left(\psi_{x}, \psi_{y}\right)_{D_{0}}\right| \leq \frac{\delta^{\prime \prime}}{d_{\min }}\left\|\psi_{x}\right\|_{D_{0}} \cdot\left\|\psi_{y}\right\|_{D_{0}}
$$

for the same $\psi_{x}, \psi_{y}$ as there. By simple standard calculations this implies that

$$
\left\|\sum_{x} \psi_{x}\right\|_{D_{0}}^{2} \geq\left(1-\frac{\delta^{\prime \prime}}{d_{\min }}(\tilde{M}-1)\right) \sum_{x}\left\|\psi_{x}\right\|_{D_{0}}^{2},
$$

where the sums are over all $x \in \mathcal{Z}$ with $\left|x-x_{0}\right| \leq \delta^{\prime}, \psi_{x} \in W(x)$ are arbitrary and $\tilde{M}$ is the number of summands. Now by A) above $\tilde{M} \leq M$. Since $\delta<\varepsilon^{\prime}$ the expression in parantheses is $\geq \frac{1}{2}$ for sufficiently small $\varepsilon^{\prime}$, so (24) gives $\sum_{x}\left\|\psi_{x}\right\|_{D_{0}}^{2} \leq$ $2\left\|\sum_{x} \psi_{x}\right\|_{D_{0}}^{2}$, which with (22) gives $\sum_{x}\left\|\psi_{x}\right\|^{2} \leq 2 \frac{d_{\max }}{d_{\min }}\left\|\sum_{x} \psi_{x}\right\|^{2}$ and so

$$
\sum_{x}\left\|\psi_{x}\right\| \leq \sqrt{2 M \frac{d_{\max }}{d_{\min }}}\left\|\sum_{x} \psi_{x}\right\| .
$$

We return to (20). If $\psi \in W^{\prime}=\bigoplus_{\left|x-x_{0}\right| \leq \delta^{\prime}} W(x), \psi=\sum_{x} \psi_{x}$, then we get from (25)

$$
\begin{aligned}
\left\|\psi-P_{\delta}\left(x_{0}\right) \psi\right\| & \leq \sum_{x}\left\|\psi_{x}-P_{\delta}\left(x_{0}\right) \psi_{x}\right\| \leq \varepsilon_{1} \sum_{x}\left\|\psi_{x}\right\| \\
& \leq \varepsilon_{2}\|\psi\|, \quad \varepsilon_{2}:=\varepsilon_{1} \sqrt{2 M \frac{d_{\max }}{d_{\min }}}
\end{aligned}
$$


This means that $W^{\prime}$ is close to $W_{0}:=\operatorname{Ran} P_{\delta}\left(x_{0}\right)$. Now by A) above, $W^{\prime}$ and $W_{0}$ have the same dimension, so this implies by standard arguments that $W_{0}$ is also close to $W^{\prime}$; more precisely, with $P_{W^{\prime}}: V \rightarrow W^{\prime}$ the orthogonal projection,

$$
\left\|\psi-P_{W^{\prime}} \psi\right\| \leq \varepsilon_{2}\|\psi\|, \quad \psi \in W_{0} .
$$

Finally, assume that $\varphi \in V$ satisfies (11). Then (15) with $\delta$ as above together with (27) give, again by standard facts about distances of subspaces,

$$
\left\|\varphi-P_{W^{\prime}} \varphi\right\| \leq\left(\varepsilon_{2}+\frac{\varepsilon}{\delta}\right)\|\varphi\| .
$$

Putting everything together, we have $\delta=\varepsilon s^{k}$ for some $k \in\{1, \ldots, M\}$ with $s=$ $\left(\varepsilon^{\prime} / \varepsilon\right)^{1 /(M+1)}$, which implies that $\varepsilon / \delta \leq s^{-1}$ and $\delta^{\prime} \leq \varepsilon^{\prime}$. Also, $\varepsilon_{1}=\frac{4}{s} \frac{d_{\max }}{d_{\min }}$ and $\varepsilon_{2}=\varepsilon_{1} \sqrt{2 M \frac{d_{\max }}{d_{\min }}}$. Altogether, the right-hand side of $(\underline{28)})$ is bounded by $C s^{-1}\|\varphi\|$, and since the left-hand side only decreases when replacing $W^{\prime}$ by the bigger space $\bigoplus_{\left|x-x_{0}\right| \leq \varepsilon^{\prime}} W(x)$, the theorem is proven.

\section{Monotonicity of $U$ And of its Logarithm}

Let $U(x)$ be a real analytic unitary family. Then there is an analytic family of symmetric operators $A(x)$ with $U(x)=e^{i A(x)}$ for all $x$ : Simply take $A(x)$ as the operator having eigenfunctions $\varphi_{j}(x)$ with eigenvalue $\mu_{j}(x)$ for $j=1, \ldots, \operatorname{dim} V$. Since monotonicity of $U$ implies monotonicity of the $\mu_{j}$, by (7), it is natural to ask whether it even implies monotonicity of $A$, i.e. $\frac{d}{d x} A(x) \geq 0$. We show that this is not true but that the opposite implication is true.

Proposition 5. Let $x \mapsto A(x)$ be a $C^{1}$ family of symmetric operators and $U(x)=$ $e^{i A(x)}$. Then

$$
\frac{1}{i} U^{\prime} U^{-1}=\int_{0}^{1} e^{i \tau A} A^{\prime} e^{-i \tau A} d \tau .
$$

Here, a prime denotes differentiation with respect to $x$, and $U, U^{\prime}, A, A^{\prime}$ are taken at a fixed $x$.

Proof. Let $W(t, x)=\frac{1}{i} \frac{\partial}{\partial x} e^{i t A(x)}$. Then

$$
\frac{\partial}{\partial t} W=\frac{1}{i} \frac{\partial}{\partial x} \frac{\partial}{\partial t} e^{i t A}=\frac{\partial}{\partial x} A e^{i t A}=A^{\prime} e^{i t A}+i A W .
$$

Now fix $x$, and let $B(t)=A^{\prime} e^{i t A}$. The solution of the ordinary differential equation $\frac{d}{d t} Y(t)=B(t)+i A Y(t)$ with $Y(0)=0$ is

$$
Y(t)=\int_{0}^{t} e^{i(t-\tau) A} B(\tau) d \tau
$$

as can be verified directly. (This is called Duhamel's principle.) Now $W(\cdot, x)$ satisfies the same first-order ODE and initial condition as $Y$, so it follows that $W(t, x)=Y(t)$ for all $t$. Now rewrite $Y(t)=\int_{0}^{t} e^{i \tau A} B(t-\tau) d \tau$ and set $t=1$ to obtain (29).

Corollary 6. Let $x \mapsto A(x)$ be a $C^{1}$ family of symmetric operators. If $A^{\prime}(x)>0$ for each $x$, then the unitary family $U(x)=e^{i A(x)}$ is monotone.

Proof. Positivity of $A^{\prime}$ implies positivity of $e^{i \tau A} A^{\prime} e^{-i \tau A}$ for each $\tau$, so the claim follows from (29). 
The converse is not true. As an example let $A_{0}=\left(\begin{array}{cc}0 & -\pi \\ \pi & 0\end{array}\right), B=\left(\begin{array}{cc}-b & 0 \\ 0 & 1\end{array}\right)$ with $0<b<1$ and $A(x)=A_{0}+x B$. Then $e^{i \tau A_{0}}=\left(\begin{array}{cc}\cos \pi \tau & -\sin \pi \tau \\ \sin \pi \tau & \cos \pi \tau\end{array}\right)$ is rotation by $\pi \tau$, and a short calculation shows that $\int_{0}^{1} e^{i \tau A_{0}} B e^{-i \tau A} d \tau=\frac{1-b}{2} I$ (this is also clear without calculation since the result must be rotation-invariant with trace equal to $\operatorname{tr} B=1-b$; in essence, the negative direction of $B$ gets averaged away against the positive direction). Therefore, $U(x)=e^{i A(x)}$ is monotone near $x=0$, but $A^{\prime}(0)=B$ is not positive.

\section{TWO-PARAMETER FAMILIES}

Theorem 7. Let $U(x, y)$ be a unitary operator in a finite-dimensional Hermitian vector space depending real analytically on $x, y \in \mathbb{R}$. Assume

$$
\frac{1}{i} \frac{\partial U}{\partial x} U^{-1}>0 \quad \text { at }\left(x_{0}, y_{0}\right)
$$

Then the set $\{(x, y): U(x, y)$ has eigenvalue one $\}$ is, in a neighborhood of $\left(x_{0}, y_{0}\right)$, a union of real analytic curves $x=x_{j}(y)$. The corresponding projections $P_{j}(y)$ to the eigenspace of $U\left(x_{j}(y), y\right)$ with eigenvalue one are also analytic functions of $y \neq y_{0}$, extending analytically to $y=y_{0}$, and $\sum_{j} P_{j}\left(y_{0}\right)$ is the projection to $\operatorname{ker}\left(I-U\left(x_{0}, y_{0}\right)\right)$.

Note that in general it is not true that the eigenvalues and eigenprojections of $U(x, y)$ may be arranged as real analytic functions of $(x, y)$; see [3, II.6.1]. As an example one may take $A(x, y)=\left(\begin{array}{cc}3 x & y \\ y & x\end{array}\right)$ and $U(x, y)=e^{i A(x, y)}$ and $\left(x_{0}, y_{0}\right)=$ $(0,0)$. The matrix $A(x, y)$ has eigenvalues $2 x \pm \sqrt{x^{2}+y^{2}}$. Clearly there are no analytic functions $\mu_{1}, \mu_{2}$ of $(x, y)$ so that $\left\{\mu_{1}(x, y), \mu_{2}(x, y)\right\}=\left\{2 x \pm \sqrt{x^{2}+y^{2}}\right\}$ for all $(x, y)$ in a neighborhood of $(0,0)$. However, the set where at least one of these eigenvalues is zero is the union of the analytic curves $x= \pm \frac{1}{\sqrt{3}} y$. The positivity assumption is also satisfied: One has $\frac{1}{i} \frac{\partial U}{\partial x} U^{-1}=\frac{\partial A(0,0)}{\partial x}=\left(\begin{array}{ll}3 & 0 \\ 0 & 1\end{array}\right)>0$ at $(0,0)$.

Note also that the statement of the theorem reduces to the well-known facts of one-parameter perturbation theory in case $U(x, y)=e^{i x} U(y)$, for an analytic one-parameter family of unitary operators $U(y)$.

Proof. Let w.l.o.g. $x_{0}=y_{0}=0$.

We first consider the case $U(0,0)=I$. Let $A=\frac{1}{i} \log U$ near $(x, y)=(0,0)$. Here $\frac{1}{i} \log$ is understood as the local inverse of the map $A \mapsto e^{i A}$, which is invertible near $A=0$ since its differential at $A=0$ is $i$ times the identity. Then the operators $A(x, y)$ are selfadjoint, $A(0,0)=0$, and $\partial A / \partial x(0,0)>0$ since it equals $\frac{1}{i} \frac{\partial U}{\partial x} U^{-1}(0,0)$ by (29), and we need to prove that the set $S=\{(x, y)$ : $A(x, y)$ is not invertible $\}$ is a union of real analytic curves as claimed 1

If $A(x, y)=x A+y B$ is linear in $x, y$, then (since $A>0$ ) $A$ and $B$ may be diagonalized simultaneously, hence may be assumed to be diagonal, and then it is obvious that $S$ is a union of lines, $x_{j}(y)=y b_{j} / a_{j}$, where $a_{j}, b_{j}$ are the diagonal entries of $A, B$, respectively. In general, write $A(x, y)=x A+y B+C(x, y)$ with

\footnotetext{
${ }^{1}$ The author is grateful to Y. Colin-de-Verdière for a fruitful discussion on this.
} 
$C(x, y)=O\left(|x, y|^{2}\right)$ and w.l.o.g. $A, B$ diagonal. Then, if the dimension of the vector space is $M$,

$$
\operatorname{det} A(x, y)=\prod_{j=1}^{M}\left(x a_{j}+y b_{j}\right)+O\left(|x, y|^{M+1}\right),
$$

and a standard argument (using polar coordinates) shows that the zero set of this function is a union of real analytic lines $x=x_{j}(y)$ having tangents $x a_{j}+y b_{j}=0$ at the origin.

If $U(0,0)$ is arbitrary, let $W=U-I$ (where $I$ denotes the identity) and $V_{0}=$ $\operatorname{Ker} W(0,0)$ and $V_{1}$ be its orthogonal complement. Let $W_{k l}(x, y), k, l=0,1$, be the 'submatrices' of $W(x, y)$ corresponding to the decomposition $V_{0} \oplus V_{1}$. Then $W_{00}$, $W_{01}$ and $W_{10}$ vanish at $(x, y)=(0,0)$, and $W_{11}$ is invertible at $(0,0)$ and hence in a neighborhood. Then the equation $W\left(v_{0} \oplus v_{1}\right)=0$, where $v_{0} \in V_{0}, v_{1} \in V_{1}$, is equivalent to $W^{\prime} v_{0}=0$, where $W^{\prime}=W_{00}-W_{01} W_{11}^{-1} W_{10}$, and $v_{1}=-W_{11}^{-1} W_{10} v_{0}$. Therefore, $U(x, y)$ has eigenvalue one iff the operator $U^{\prime}(x, y)=W^{\prime}(x, y)+I_{V_{0}}$ on $V_{0}$ has eigenvalue one. One easily checks that $U^{\prime}(x, y)$ is unitary. Since $W^{\prime}(0,0)=0$ the claim now follows from the case considered first.

Let $C_{j}(y)=U\left(x_{j}(y), y\right)$ and let $P_{j}(y)$ be the projection to $\operatorname{Ker} C_{j}(y)$. Since $C_{j}$ is analytic in $y$, its eigenprojections are analytic for $y \neq 0$ (but near zero) and extend analytically to $y=0$ (see [3]), so this is in particular true for $P_{j}$.

\section{REFERENCES}

[1] S. Gnutzmann and U. Smilansky, Quantum graphs: Applications to quantum chaos and universal spectral statistics, Advances in Physics 55 (2006), no. 5-6, 527-625.

[2] Daniel Grieser, Spectra of graph neighborhoods and scattering, Proceedings of the London Mathematical Society 97 (2008), 718-752. MR2448245 (2011c:58059)

[3] Tosio Kato, Perturbation theory for linear operators. 2nd ed., Grundlehren der mathematischen Wissenschaften. 132. Berlin-Heidelberg-New York: Springer-Verlag, 1976. MR.0407617 (53:11389)

[4] Franz Rellich, Störungstheorie der Spektralzerlegung. I. Analytische Störung der isolierten Punkteigenwerte eines beschränkten Operators, Math. Ann. 113 (1937), 600-619 (German). MR 1513109

Institut für Mathematik, Carl von Ossietzky Universität Oldenburg, D-26111 OldENBURG, GERMANY

E-mail address: daniel.grieser@uni-oldenburg.de 